\title{
Biodegradable microfibrillar polymer-polymer composites from poly(L-lactic acid)/poly(glycolic acid)
}

\author{
L. D. Kimble*, D. Bhattacharyya, S. Fakirov
}

The Centre for Advanced Composite Materials, The University of Auckland, Building 740, Room 181, Tamaki Campus, 261 Morrin Rd, Glen Innes, Auckland, New Zealand

Received 1 October 2014; accepted in revised form 6 December 2014

\begin{abstract}
Biodegradable coronary stents have been under development for several years and a trend in biodegradable stent material development has emerged: reinforcement to enhance mechanical properties and creep resistance to improve vessel support. The aim of this work is to investigate the mechanical and viscoelastic characteristics of poly(L-lactic acid)/poly (glycolic acid) (PLLA/PGA) microfibrillar polymer-polymer composites (MFCs) at $37^{\circ} \mathrm{C}$ to determine the suitability of PGA fibrils as a reinforcement for polymeric, biodegradable stents. PLLA/PGA MFCs were produced via cold-drawing and subsequent compression moulding of extruded PLLA/PGA blend wires. Scanning electron microscopy revealed excellent fibril formation in the case of a 70/30 wt $\%$ PLLA/PGA MFC- the mean fibril diameter being $400 \mathrm{~nm}$ and aspect ratios exceeding 250. Tensile tests demonstrate Young's modulus and strength increases of 35 and $84 \%$ over neat PLLA in the case of a 70/30 wt\% PLLA/PGA MFC. Creep resistance of the PLLA/PGA MFCs is lower than that of neat PLLA, as shown via relaxation. Dynamic mechanical thermal analysis demonstrates that it is the onset of glass transition of PGA that is the underlying cause for low creep resistance of the PLLA/PGA MFCs at $37^{\circ} \mathrm{C}$.
\end{abstract}

Keywords: biocompatible polymers, biodegradable polymers, mechanical properties, microfibrillar polymer-polymer composites, viscoelasticity

\section{Introduction}

Stents have been used to treat coronary artery disease (the build-up of plaque in coronary arteries) for decades and they have evolved significantly. The stenting process begins with balloon angioplasty during which a balloon catheter is navigated to the target site and inflated to widen the vessel and flatten the plaque, as illustrated in Figure 1a-1c. A second balloon catheter with a stent over the balloon is then navigated to the target site and deployed via balloon expansion, Figure 1d-1e). Finally the balloon is deflated and removed, leaving the stent in place to support the vessel, Figure 1f-1g.

Early stents were made of medical grade stainless steel [1] and are known as bare metal stents (BMSs). Unfortunately the occurrence of restenosis (re-nar- rowing of the vessel via either an inflammatory reaction [2] or formation of a clot/thrombus [3]) was common after BMS implantation. For this reason attempts were made at coating stents with various compounds, e.g. gold, silicon carbide, titaniumnitride-oxide, etc. to make them more inert $[3,4]$. These stents are known as coated metal stents (CMSs); their efficacy results were mixed - in some cases yielding higher restenosis rates than BMSs [5]. The next step in stent evolution was the development of drug-eluting stents (DESs) which gradually release pharmaceutical agents after implantation to mitigate restenosis. However, clinical studies revealed that late stent thrombosis rates were higher in the case of DESs when compared with

\footnotetext{
*Corresponding author, e-mail: 1kim009@aucklanduni.ac.nz (C) BME-PT
} 


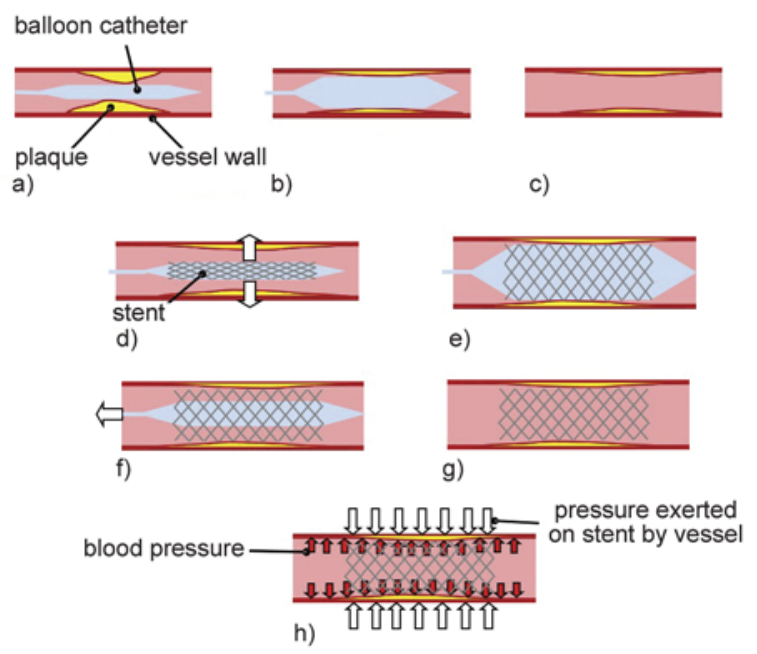

Figure 1. Stenting process: balloon catheter navigated to target site (a), inflation of balloon to deform plaque (b), vessel after removal of the balloon catheter (c), stent on balloon catheter is navigated to target site and deployed via balloon expansion (d-e), balloon is deflated and removed, leaving the stent in place to support the vessel $(\mathrm{f}-\mathrm{g})$. The stent is under continuous crushing load exerted by the vessel (h).

BMSs [6] and this has been attributed to endothelialisation being delayed by the eluted drugs [7]. Cardiologists have noted that vessel support is required for only 6 months while they heal [8]. For this reason biodegradable stents were developed which perform the required role and eventually degrade entirely, leaving no permanent object in the patients' bodies. Poly(L-lactic acid), PLLA, is a very prominent material in biodegradable stent material development but its major disadvantage is that it is brittle [9]. Ductility is required during balloon expansion in order for the stent to undergo sufficient plastic deformation without fracturing [10]. It is possible to modify PLLA via plasticiser addition or blending with rubbery polymers to produce a ductile material. Grabow and coworkers [10-12] have blended PLLA with tri-ethyl citrate (TEC), poly( $\varepsilon$-caprolactone) (PCL) and poly(4-hydroxybutyrate) (P4HB) for the intended application as biodegradable stent materials. Significant reduction in elastic moduli resulted ( $\sim 50 \%$ in the cases of PLLA/ P4HB and PLLA/PCL/TEC blends $[11,12]$ and creep resistance of PLLA/TEC was reported to have greatly reduced by $1-2$ orders of magnitude [10]. Blends of PLLA and poly(butylene succinate) (PBS) have been investigated by the authors and although they exhibit favourable mechanical properties, creep resistance reduces rapidly during degradation [13].
Creep resistance must be maintained because a stent is subjected to radial pressure by the vessel it supports and insufficient creep resistance could result in stent collapse or narrowing. Therefore, there is a need to investigate means of reinforcing polymeric biodegradable stent materials. Further motivation comes from evidence that the industry is moving in this direction - medical device companies have, in the last few years, published patents on reinforcement of biodegradable, polymeric stents. The patents cover the use of mineral reinforcements such as nanoclays $[14,15]$.

A common method of reinforcing polymers is to use particulate reinforcements such as nanoclays, a method which is widely used already. Furthermore, this method is covered by the aforementioned patents on reinforcement of biodegradable, polymeric stents $[14,15]$. An alternative method is the use of microfibrillar or nanofibrillar polymer-polymer composites (MFCs or NFCs, respectively). The concept was developed by Fakirov et al. [16] with the knowledge that drawing of polymers enhances their mechanical properties. These polymer-polymer composites are created by fibrillising the dispersed component of an immiscible polymer blend, often by drawing during which the dispersed particles elongate and coalesce to form fibrils. The final step is to isotropise the matrix polymer by heating the material above the melting point of the matrix but below that of the dispersed polymer [17-22]. The MFC process has been shown to be successful for petrochemical-derived polymer pairs, including poly(ethylene terephthalate)/low-density poly(ethylene) [20], poly(propylene)/PET [22] and single polymer composites of polyamide 6 [23] with properties such as modulus and strength increasing manifold when compared with the neat, isotropic matrix polymer. Little work has been done on biodegradable, biocompatible MFCs or NFCs so there is enormous scope for research in this area for biomedical applications, as well as packaging or other consumer-oriented applications. Friedrich et al. [24] report briefly on MFCs from PLLA and poly(glycolic acid), PGA. They report improved bending modulus and strength compared with neat PLLA but importantly, their results were obtained at room temperature - despite the intended application being bone nails [24]. The mechanical characteristics of polymers are highly dependent on temperature, therefore for biomedical applications the properties should be measured at $37^{\circ} \mathrm{C}$. 
The aim of this work is to determine the mechanical properties and viscoelastic behaviour of PLLA/PGA MFCs at $37^{\circ} \mathrm{C}$ in order to assess the suitability of PGA fibrils for reinforcement of biodegradable stents based on PLLA.

\section{Experimental methodology}

\subsection{Materials}

PLLA grade 2002D (NatureWorks LLC, USA) was supplied in pellet form. Manufacturer's data indicates that the PLLA has a density of $1.24 \mathrm{~g} / \mathrm{cm}^{3}$ and a melt flow index of $4-8 \mathrm{~g} / 10 \mathrm{~min}\left(190^{\circ} \mathrm{C}, 2.16 \mathrm{~kg}\right)$. Its glass transition temperature $\left(T_{\mathrm{g}}\right)$ is $55-60^{\circ} \mathrm{C}$ and melting temperature, $T_{\mathrm{m}}$, is $180^{\circ} \mathrm{C}$ according to Letchford et al. [25]. PGA (Kuredux, Kureha Corporation, Japan) was also supplied in pellet form. It is indicated by the manufacturer to have a $T_{\mathrm{m}}$ of $220^{\circ} \mathrm{C}$, $T_{\mathrm{g}}$ of $40^{\circ} \mathrm{C}$ and density of $1.5-1.6 \mathrm{~g} / \mathrm{cm}^{3}$, depending on the degree of crystallinity.

\subsection{Specimen preparation}

PLLA and PGA were dried overnight at 70 and $120^{\circ} \mathrm{C}$, respectively, before melt blending in a twinscrew extruder (DSE 25, Brabender, Germany) at PLLA/PGA wt $\%$ ratios $70 / 30$ and 80/20 - extrudate was collected on a spool as a wire. The temperature profile used for blending is shown in Table 1.

The extruded wire was cold-drawn (i.e. below the $T_{\mathrm{m}}$ but above the $T_{\mathrm{g}}$ of the PLLA matrix) at $60^{\circ} \mathrm{C}$ such that drawing occurred via necking. The drawing equipment was custom made at our facility and consists of a heated channel with rollers on either side. The rollers at the end of the channel were set to rotate faster than those feeding the wire in at the start of the channel. This provided the tension necessary for drawing. The MFCs were produced via compression moulding (using a hot press, Carver, USA) of drawn wire which had been wound around

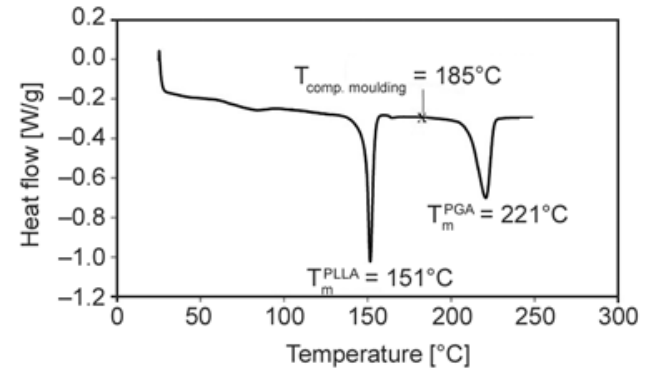

Figure 2. DSC scan of drawn PLLA/PGA30

a teflon-covered aluminum plate. Moulding was done at $T_{\text {comp.moulding }}=185^{\circ} \mathrm{C}$ since $T_{\mathrm{m}, \mathrm{PLLA}}<$ $185^{\circ} \mathrm{C}<T_{\mathrm{m}, \mathrm{PGA}}$ as determined by differential scanning calorimetry (DSC), Figure 2. A DSC Q2000 (TA Instruments, USA) was used - a temperature ramp rate of $10^{\circ} \mathrm{C} / \mathrm{min}$ was used.

The resulting unidirectional PLLA/PGA MFCs were cut into rectangular strips $150 \mathrm{~mm} \times 14 \mathrm{~mm}$ with thicknesses $\sim 0.4 \mathrm{~mm}$ with the length parallel with the fiber direction for tensile and relaxation tests. Specimens from as-extruded blends were prepared in the same way and nomenclature of all specimen types is provided in Table 2).

\subsection{Tensile and relaxation tests}

A universal testing machine (5567, Instron, USA) was used for tensile and relaxation tests - all of which were performed in an environmental chamber at $37^{\circ} \mathrm{C}$. Strain of a $50 \mathrm{~mm}$ gauge length marked on each specimen was measured using a video extensometer (Advanced Video Extensometer, Instron, USA). Tensile tests were performed in accordance with ASTM D882 - distance between the grips: $100 \mathrm{~mm}$, crosshead speed: $10 \mathrm{~mm} / \mathrm{min} .5$ specimens of each material were tensile tested. Young's moduli were determined using the gradient of the stressstrain data between 0.2 and $0.5 \%$ strain to mitigate the influence of the curves' toes. Relaxation tests

Table 1. Extrusion temperature profile for blending PLLA and PGA

\begin{tabular}{|c|c|c|c|c|c|c|c|c|c|}
\hline Zone & $\mathbf{1}$ & $\mathbf{2}$ & $\mathbf{3}$ & $\mathbf{4}$ & $\mathbf{5}$ & $\mathbf{6}$ & $\mathbf{7}$ & $\mathbf{8}$ & DIE \\
\hline Temperature $\left[{ }^{\circ} \mathrm{C}\right]$ & 190 & 190 & 220 & 230 & 235 & 230 & 220 & 215 & 210 \\
\hline
\end{tabular}

Table 2. Nomenclatures of PLLA/PGA blends and associated MFCs

\begin{tabular}{|l|c|l|}
\hline \multicolumn{1}{|c|}{ Description } & $\begin{array}{c}\text { PLLA/PGA ratio } \\
{[\mathbf{w t} \mathbf{\%} / \mathbf{w t} \%]}\end{array}$ & \multicolumn{1}{|c|}{ Designation } \\
\hline \multirow{2}{*}{ PLLA/PGA blend, not drawn } & $80 / 20$ & PLLA/PGA20 \\
\cline { 2 - 3 } PLLA/PGA MFC resulting from compression moulding of drawn wire & $70 / 30$ & PLLA/PGA30 \\
\cline { 2 - 3 } & $80 / 20$ & PLLA/PGA20-MFC \\
\hline
\end{tabular}


were done by applying $20 \mathrm{MPa}$ initial stress and fixing the extension then monitoring stress over 15 mins.

\subsection{Viscoelastic characterisation}

Rectangular specimens $40 \mathrm{~mm} \times 5 \mathrm{~mm}$ were cut from compression moulded films of PLLA/PGA20MFC, PLLA/PGA30-MFC and neat PLLA. Tensile mode dynamic mechanical thermal analysis (DMTA), with a strain amplitude of $0.05 \%$ and frequency of $1 \mathrm{~Hz}$ was done using a DMA Q800 (TA Instruments, USA).

\subsection{Morphological characterisation}

Scanning electron microscopy (SEM) was used to observe the morphology of PLLA/PGA blends and their corresponding MFCs. Specimens were prepared via cryofracture. Additional specimens were prepared via selective solvent extraction in a Soxhlet apparatus using tetrahydrofuran as the selective solvent. This resulted in the PLLA matrix being removed, leaving PGA fibrils behind. All specimens were coated with platinum before observation. A Philips (Netherlands), model XL30 S-FEG, scanning electron microscope was used.

\section{Results and discussion}

\subsection{Morphology}

SEM images of PLLA/PGA30 and PLLA/PGA20 demonstrate that in both cases PGA is very well dispersed within the PLLA, as shown in Figure $3 \mathrm{a}$ and $3 \mathrm{~b}$, respectively. PGA particle diameters are approximately $1-2 \mu \mathrm{m}$ in diameter. In the case of PLLA/ PGA30 there is a higher frequency of closely-clustered PGA particles compared with that observed in PLLA/PGA20. If one observes film made from non-drawn PLLA/PGA, fractured parallel to the direction of the wire before hot pressing, one may observe cigar shaped PGA particles, Figure $3 \mathrm{e}$ and $3 \mathrm{f}$. This shows that during production of the wire there is some degree of hot drawing occurring during haul-off of the extrudate from the extruder. The materials produced by drawing and subsequent compression moulding were fractured parallel to the drawing direction for observation. It is clear from Figure $3 \mathrm{e}$ and $3 \mathrm{f}$ that fibrillisation of the dispersed PGA component has occurred. Note the reduction in diameter of the cigars present in the non-drawn wire $(\sim 1 \mu \mathrm{m})$ after fibrillisation during cold drawing $(\sim 400 \mathrm{~nm})$. In the case of PLLA/PGA30-MFC, fibril formation is excellent and the PGA exists as long, well-oriented fibrils, Figure 3c. The morphology PLLA/PGA20-MFC, however, shows that drawing of PLLA/PGA20 only partially fibrillised the PGA, Figure 3d. Some fibrils can be seen but a significant proportion of the PGA present in PLLA/ PGA20-MFC still exists as round particles. This poor fibrillisation is attributed to the concentration of PGA in PLLA/PGA20 being too low, therefore the coalescence of PGA particles during drawing is hindered. Coalescence is essential for the formation of high aspect-ratio fibrils [26]. In order to determine key dimensions, fibrils were extracted from PLLA/PGA30-MFC using selective solvent extraction (tetrahydrofuran used as selective solvent) and are shown in Figure 3g and 3h. From these images and others a mean fibril diameter of $400 \mathrm{~nm}$ was determined. Fibrils exceeding $100 \mu \mathrm{m}$ in length can be observed in Figure 3e and therefore fibrils with aspect ratios greater than 250 were created. The small diameters of the fibrils makes them well-suited to reinforcement of biodegradable stents since stent struts are only $150 \mu \mathrm{m}$ thick, making some particulate reinforcements unsuitable since they have sizes of similar orders of magnitude.

\subsection{Tensile properties}

Properties of PLLA/PGA MFCs are compared with those of neat PLLA and the simple (not drawn) PLLA/PGA blends from which they were derived. It is important to make both comparisons because (i) PLLA is what is being reinforced whilst (ii) we must assess the gains obtained through MFC processing. Results from tensile testing are summarised in Table 3. Young's moduli of PLLA/PGA20 and PLLA/PGA30 are 5 and $16 \%$ higher than that of neat PLLA. The MFCs, PLLA/PGA20-MFC and PLLA/ PGA30-MFC exhibited Young's moduli 15 and 36\% higher than that of neat PLLA - clearly showing the enhancement of stiffness brought about by fibrillising the PGA, despite poor fibrillisation in PLLA/ PGA20-MFC. Note also that the PLLA/PGA blends are very brittle whilst the PLLA/PGA MFCs exhibit excellent toughness, as summarised by strains at break in Table 3 and which can be observed in stress-strain plots in Figure 4.

The enhancements of strength provided by the MFC process are evident- PLLA/PGA30-MFC exhibits a strength of $88.1 \mathrm{MPa}: 84$ and $69 \%$ higher than those of neat PLLA and PLLA/PGA30, respectively. The 

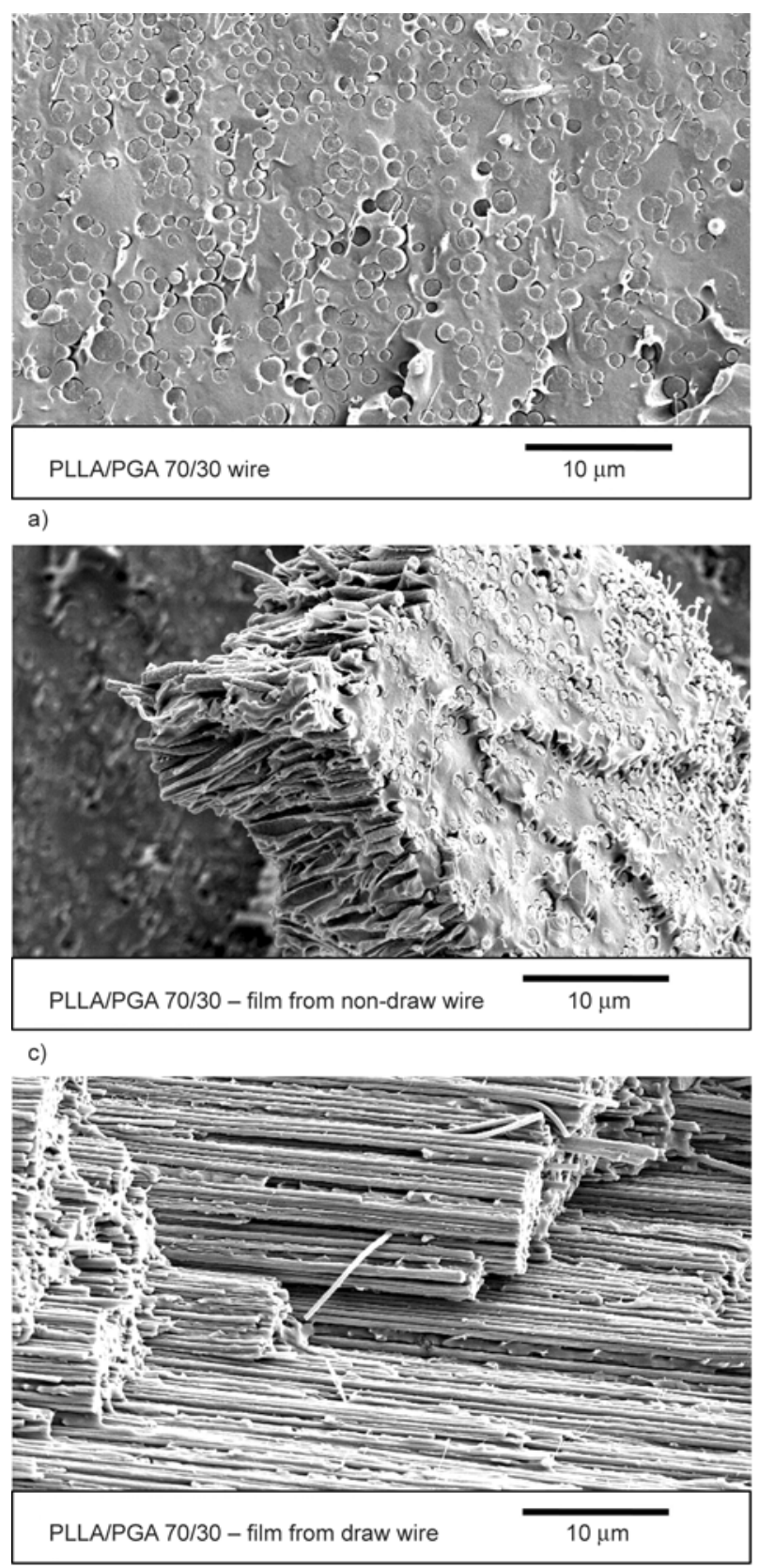

e)

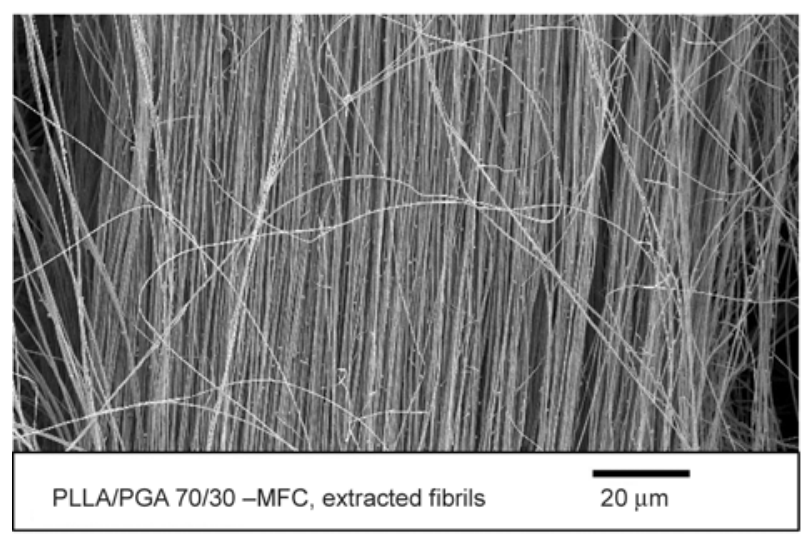

g)

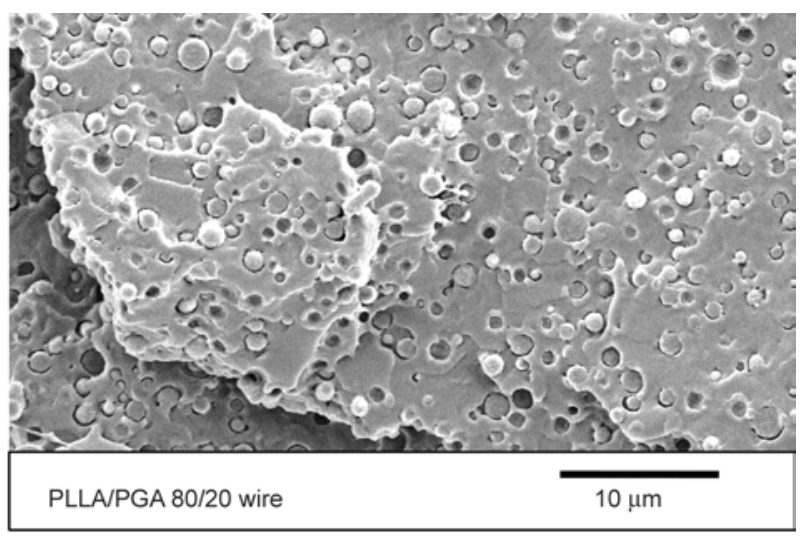

b)

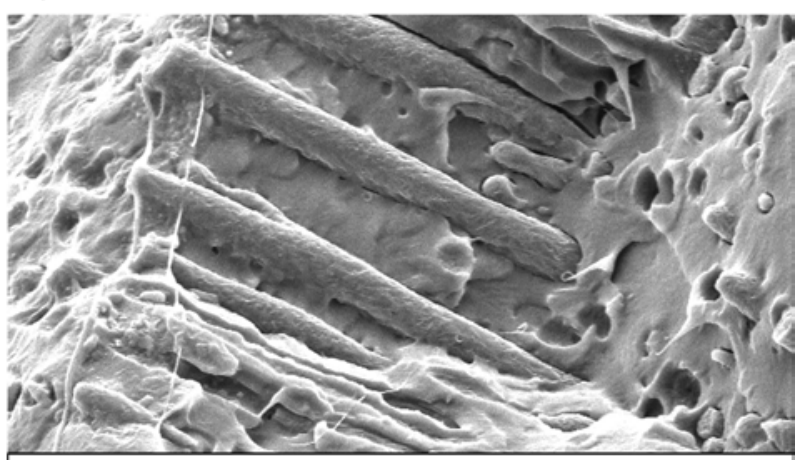

PLLA/PGA 80/20 - film from non-draw wire

$5 \mu \mathrm{m}$ d)

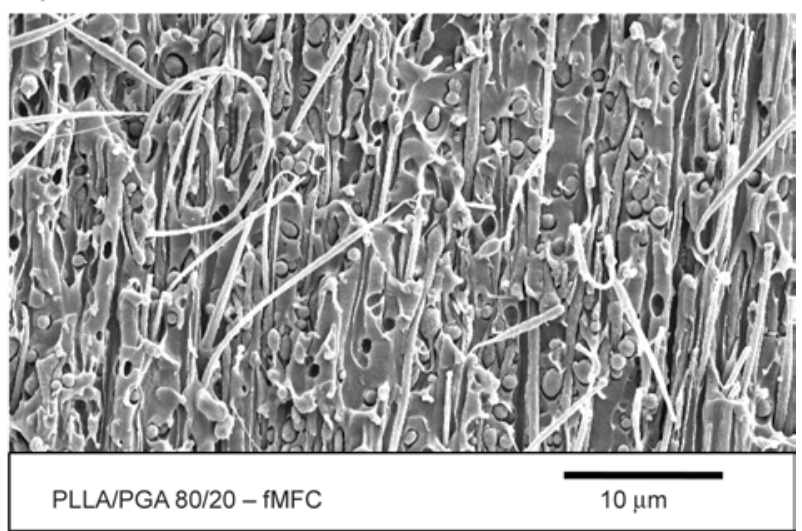

f)

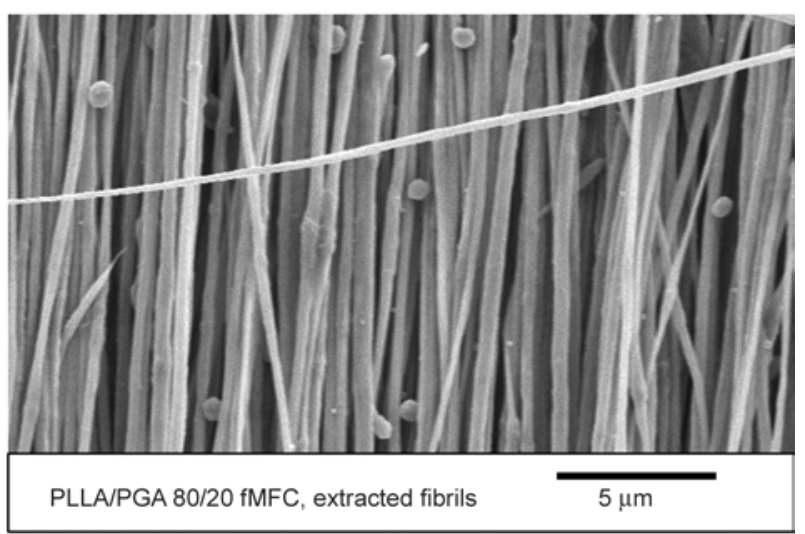

h)

Figure 3. SEM images of non-drawn PLLA/PGA20 and PLLA/PGA30 (a and b) wires and film made from non-drawn wires ( $c$ and d). The MFCs resulting from drawing and subsequent compression moulding are shown (e and f). Fibrils extracted from PLLA/PGA30-MFC (g) and example diameter measurements of them are shown (h). 

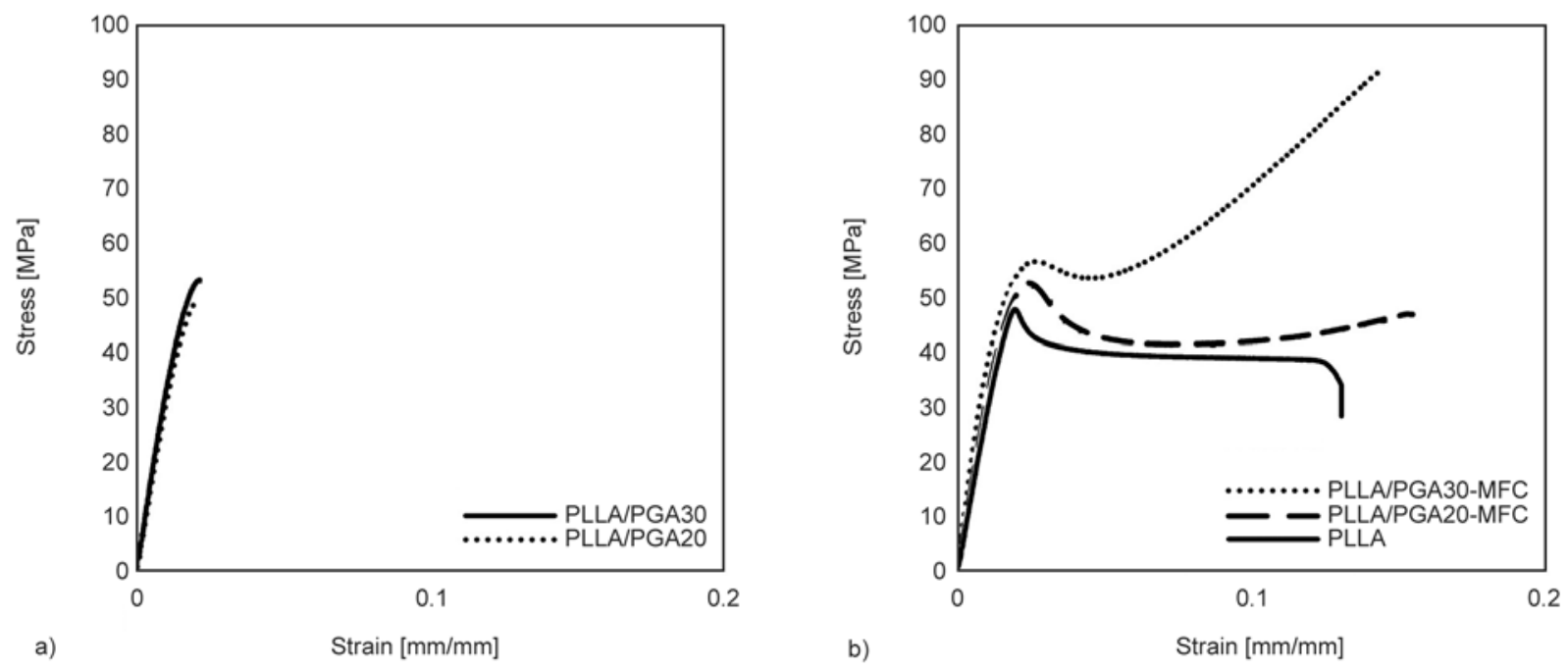

Figure 4. Stress-strain plots of PLLA/PGA blends (a) and their corresponding MFCs and neat PLLA (b)

Table 3. Strengths, Young's moduli and strains at break of PLLA, PLLA/PGA blends and their corresponding MFCs

\begin{tabular}{|l|c|c|c|}
\hline \multicolumn{1}{|c|}{ Material } & $\begin{array}{c}\text { Young's } \\
\text { modulus } \\
{[\text { GPa] }}\end{array}$ & $\begin{array}{c}\text { Tensile } \\
\text { strength } \\
{[\text { MPa] }}\end{array}$ & $\begin{array}{c}\text { Strain at } \\
\text { break } \\
{[\%]}\end{array}$ \\
\hline PLLA & $3.00 \pm 0.06$ & $48.0 \pm 3.8$ & $12.6^{*}$ \\
\hline PLLA/PGA20 & $3.16 \pm 0.07$ & $51.5 \pm 1.6$ & $2.2 \pm 0.2$ \\
\hline PLLA/PGA30 & $3.47 \pm 0.11$ & $52.0 \pm 1.8$ & $2.2 \pm 0.3$ \\
\hline PLLA/PGA20-MFC & $3.44 \pm 0.14$ & $52.5 \pm 0.6$ & $15.3 \pm 1.1$ \\
\hline PLLA/PGA30-MFC & $4.08 \pm 0.14$ & $88.1 \pm 6.8$ & $13.7 \pm 1.2$ \\
\hline
\end{tabular}

*strain at break of the one PLLA specimen which failed within the gauge length, others failed at or near the grips.

lack of improvement of strength in the case of PLLA/PGA20-MFC versus its simple blend is again attributed to poor fibrillisation, as observed by SEM, Figure $3 \mathrm{~d}$. The results of tensile testing demonstrate the successful improvement of mechanical properties of PLLA by microfibrillar reinforcement with PGA. However, the instantaneous response does not elucidate the behaviour of the material under long-term load. The results of relaxation tests and viscoelastic behaviour are now discussed to gain insight into this.

\subsection{Relaxation behaviour}

Results from relaxation tests of PLLA and PLLA/ PGA MFCs show that both PLLA/PGA20-MFC and PLLA/PGA30-MFC exhibit quicker relaxation than neat PLLA does, Figure 5. Over a 1200 s duration, PLLA specimens relaxed from an initial stress of $20 \mathrm{MPa}$ to 13-14 MPa whilst all of the PLLA/PGA MFC specimens relaxed to $10-11 \mathrm{MPa}$. Since quicker relaxation indicates lower creep resistance $[27,28]$ it can be concluded that the creep resist-

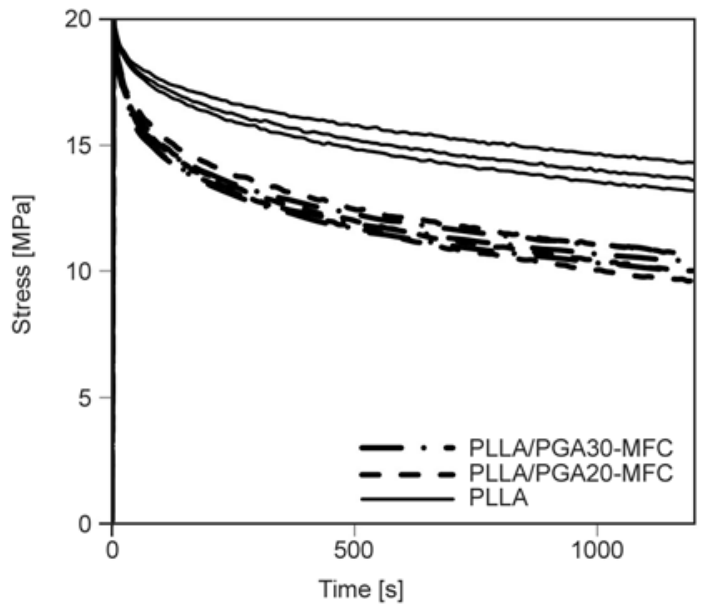

Figure 5. Stress-time curves from relaxation tests of PLLA, PLLA/PGA20-MFC and PLLA/PGA30-MFC specimens at $37^{\circ} \mathrm{C}$

ances of PLLA/PGA MFCs are lower than that of neat PLLA. This is unfavourable for reinforcement of PLLA-based stents where sufficient creep resistance is required to provide vessel support for 6 months. The thermal history of all of the specimens is very similar, therefore physical ageing may be ruled out as a potential factor affecting the creep resistance of PLLA and the PLLA/PGA MFC specimens tested. A potential cause for the lower creep resistance of the PLLA/PGA MFCs is that $37^{\circ} \mathrm{C}$ is near the $T_{\mathrm{g}}$ of PGA - for this reason DMTA was used for further investigation, as discussed in the next section.

\subsection{Viscoelastic behaviour}

In order to verify the assertion that the onset of glass transition of the amorphous phase of PGA is the cause of the creep propensity of PLLA/PGA MFCs, 

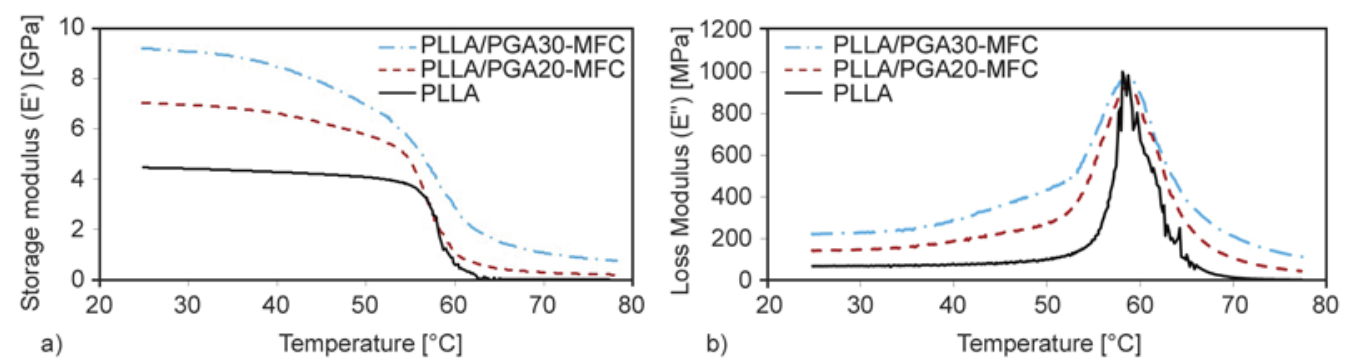

Figure 6. Storage (a) and loss moduli (b) of PLLA, PLLA/PGA20-MFC and PLLA/PGA30-MFC

their viscoelastic behaviours were compared to that of PLLA via DMTA. The storage modulus of PLLA begins to drop off at $50-55^{\circ} \mathrm{C}$ whilst those of the MFCs start to show this decline at much lower temperatures near $35^{\circ} \mathrm{C}$, especially clear in the case of PLLA/PGA30-MFC, Figure 6a. The loss moduli, which indicate energy dissipated by viscoelastic effects, echo these trends, Figure $6 \mathrm{~b}$. The more rapid increases in loss moduli of the MFCs implies that from $\sim 35^{\circ} \mathrm{C}$ the amorphous phase of PGA is becoming mobile resulting in more energy dissipation and less energy storage (as indicated by the declines in storage moduli). These results are an important consideration for load-bearing implants where creep failure is a concern. Nevertheless, other applications may benefit from the presence of PGA fibrils - note that the storage modulus of PLLA is practically negligible above $65^{\circ} \mathrm{C}$ whilst that of PLLA/ PGA30-MFC is $\sim 700 \mathrm{MPa}$ because of the crystalline phase of PGA. Some applications, such as biodegradable cups for hot beverages, for example could take advantage of this. Additional drawing to increase the orientation of PGA molecules in the fibrils could be investigated to determine whether creep resistance and mechanical properties can be improved.

\section{Conclusions}

PGA was successfully fibrillised in a PLLA matrix via cold-drawing of extruded wires of PLLA/PGA blends. Fibril formation was excellent in the case of PLLA/PGA30-MFC whilst poor fibril formation was evident in PLLA/PGA20-MFC, owing to the lower concentration of PGA preventing coalescence during drawing. Tensile tests revealed the reinforcement gained via the MFC process. PLLA/PGA30-MFC has a $35 \%$ greater Young's modulus and is $84 \%$ stronger than neat PLLA in tension at $37^{\circ} \mathrm{C}$. Relaxation tests at the same temperature indicate, however, that the creep resistance of PLLA/PGA MFCs is poorer than that of neat PLLA. DMTA results reveal that it is the onset of glass transition of PGA which is the underlying cause for the creep propensity of PLLA/PGA MFCs. For biodegradable PLLAbased stents this highlights creep failure as a significant concern. Nevertheless, the fully biodegradable MFCs produced in this work exhibit properties which may be favourable for other applications. Furthermore, there is scope for further development of PLLA/PGA MFCs. Reduction of the initial PGA particle size and/or further drawing may be used to increase orientation of PGA molecules in an attempt to enhance creep resistance. Additionally, since PLLA and PGA are both condensation polymers the possibility of transreactions between them exists. This phenomenon could be leveraged to enhance interfacial adhesion between fibrils and matrix to improve mechanical performance [23].

\section{Acknowledgements}

The authors would like to thank the University of Auckland Doctoral Scholarship and the Ministry of Business, Innovation and Employment for funding this research.

\section{References}

[1] Mani G., Feldman M. D., Patel D., Agrawal C.: Coronary stents: A materials perspective. Biomaterials, 28, 1689-1710 (2007).

DOI: 10.1016/j.biomaterials.2006.11.042

[2] Kraitzer A., Kloog Y., Zilberman M.: Approaches for prevention of restenosis. Journal of Biomedical Materials Research Part B: Applied Biomaterials, 85, 583603 (2008). DOI: $10.1002 / \mathrm{jbm} . b .30974$

[3] Serruys P. W., Kutryk M. J. B., Ong A. T. L.: Coronaryartery stents. New England Journal of Medicine, 354, 483-495 (2006). DOI: 10.1056/NEJMra051091

[4] O'brien B., Carroll W.: The evolution of cardiovascular stent materials and surfaces in response to clinical drivers: A review. Acta Biomaterialia, 5, 945-958 (2009). DOI: $10.1016 /$ j.actbio.2008.11.012 
[5] Kastrati A., Schömig A., Dirschinger J., Mehilli J., von Welser N., Pache J., Schühlen H., Schilling T., Schmitt C., Neumann F-J.: Increased risk of restenosis after placement of gold-coated stents results of a randomized trial comparing gold-coated with uncoated steel stents in patients with coronary artery disease. Circulation, 101, 2478-2483 (2000).

DOI: 10.1161/01.CIR.101.21.2478

[6] Webster M. W. I., Ormiston J. A.: Drug-eluting stents and late stent thrombosis. Lancet, 370, 914-915 (2007). DOI: $10.1016 / \mathrm{s} 0140-6736(07) 61424-\mathrm{x}$

[7] Finn A., Joner M., Nakazawa G., Kolodgie F., Newell J., John M. C., Gold H. K., Virmani R.: Pathological correlates of late drug-eluting stent thrombosis strut coverage as a marker of endothelialization. Circulation, 115, 2435-2441 (2007).

DOI: 10.1161/circulationaha.107.693739

[8] Ormiston J., Webster M.: Absorbable coronary stents. Lancet, 369, 1839-1840 (2007). DOI: $10.1016 / \mathrm{s} 0140-6736(07) 60829-0$

[9] Yokohara T., Yamaguchi M.: Structure and properties for biomass-based polyester blends of PLA and PBS. European Polymer Journal, 44, 677-685 (2008). DOI: $10.1016 /$ j.eurpolymj.2008.01.008

[10] Grabow N., Schlun M., Sternberg K., Hakansson N., Kramer S., Schmitz K-P.: Mechanical properties of laser cut poly(l-lactide) micro-specimens: Implications for stent design, manufacture, and sterilization. Journal of Biomechanical Engineering, 127, 25-31 (2005). DOI: $10.1115 / 1.1835349$

[11] Grabow N., Bünger C. M., Steinberg K., Mews S., Schmohl K., Schmitz K.: Mechanical properties of a biodegradable balloon-expandable stent from poly(1lactide) for peripheral vascular applications. Journal of Medical Devices, 1, 84-88 (2007).

DOI: $10.1115 / 1.2355683$

[12] Grabow N., Bünger C. M., Schultze C., Schmohl K., Martin D. P., Williams S. F., Sternberg K., Schmitz KP.: A biodegradable slotted tube stent based on poly(1lactide) and poly(4-hydroxybutyrate) for rapid balloonexpansion. Annals of Biomedical Engineering, 35, 2031-2038 (2007).

DOI: $10.1007 / \mathrm{s} 10439-007-9376-9$

[13] Kimble L., Bhattacharyya D.: In vitro degradation effects on strength, stiffness, and creep of PLLA/PBS: A potential stent material. International Journal of Polymeric Materials and Polymeric Biomaterials, 64, 299310 (2014).

DOI: $10.1080 / 00914037.2014 .945203$

[14] Wang Y.: Bioabsorbable scaffolds made from composites. U.S. Patent 20120290073 A1, USA (2012).

[15] Wu T.: Biodegradable stent formed with polymer-bioceramic nanoparticle composite and preparation method thereof. WO 2012100651 A1 (2012).

[16] Fakirov S., Evstatiev M., Schultz J. M.: Microfibrillar reinforced composite from drawn poly(ethylene terephthalate)/nylon-6 blend. Polymer, 34, 4669-4679 (1993). DOI: $10.1016 / 0032-3861(93) 90700-\mathrm{K}$
[17] Fakirov S., Evstatiev M.: Microfibrillar reinforced composites - New materials from polymer blends. Advanced Materials, 6, 395-398 (1994). DOI: 10.1002 /adma.19940060513

[18] Kargin V., Bakeev N., Fakirov S.: New direct observation technique of structure of polymer solutions with aid of electron microscope (in Russian). Doklady Akademii Nauk SSSR, 159, 885-888 (1964).

[19] Apostolov A. A., Fakirov S.: Effect of the block length on the deformation behavior of polyetheresters as revealed by small-angle X-ray scattering. Journal of Macromolecular Science Part B: Physics, 31, 329-355 (1992).

DOI: $10.1080 / 00222349208215520$

[20] Fakirov S., Kamo H., Evstatiev M., Friedrich K.: Microfibrillar reinforced composites from PET/LDPE blends: Morphology and mechanical properties. Journal of Macromolecular Science Part B: Physics, 43, 775-789 (2004). DOI: $10.1081 / \mathrm{mb}-120030024$

[21] Fakirov S., Bhattacharyya D., Shields R. J.: Nanofibril reinforced composites from polymer blends. Colloids and Surfaces A: Physicochemical and Engineering Aspects, 313-314, 2-8 (2008).

DOI: $10.1016 /$ j.colsurfa.2007.05.038

[22] Fakirov S., Bhattacharyya D., Lin R. J. T., Fuchs C., Friedrich K.: Contribution of coalescence to microfibril formation in polymer blends during cold drawing. Journal of Macromolecular Science Part B: Physics, 46, 183-194 (2007).

DOI: $10.1080 / 00222340601044375$

[23] Bhattacharyya D., Maitrot P., Fakirov S.: Polyamide 6 single polymer composites. Express Polymer Letters, 3, 525-532 (2009).

DOI: $10.3144 /$ expresspolymlett.2009.65

[24] Friedrich K., Hoffmann J., Evstatiev M., Ye L., Mai Y. W.: Improvements of stiffness and strength of bioresorbable bone nails by the MFC-concept. Key Engineering Materials, 334-335, 1181-1184 (2007). DOI: 10.4028/www.scientific.net/KEM.334-335.1181

[25] Letchford K., Sodegraad A., Plackett D., Gilchrist S., Burt H.: Lactide and glycolide polymers. in 'Biodegradable polymers in clinical use and clinical development' (eds.: Domb A., Kumar N., Azra A.) Wiley, Chichester, 319-365 (2011).

[26] Friedrich K., Evstatiev M., Fakirov S., Evstatiev O., Ishii M., Harrass M.: Microfibrillar reinforced composites from PET/PP blends: Processing, morphology and mechanical properties. Composites Science and Technology, 65, 107-116 (2005). DOI: 10.1016/j.compscitech.2004.06.008

[27] Aklonis J. J., Tobolsky A. V.: Relationship between creep and stress relaxation. Journal of Applied Physics, 37, 1949-1952 (1966).

DOI: $10.1063 / 1.1708645$

[28] Siengchin S., Psarras G. C., Karger-Kocsis J.: POM/ $\mathrm{PU} /$ carbon nanofiber composites produced by watermediated melt compounding: Structure, thermomechanical and dielectrical properties. Journal of Applied Polymer Science, 117, 1804-1812 (2010).

DOI: 10.1002/app.32133 